Boletín de la Sociedad Geológica Mexicana

VOLUMEN 68, NÚM. 2, 2016, P. 187-197

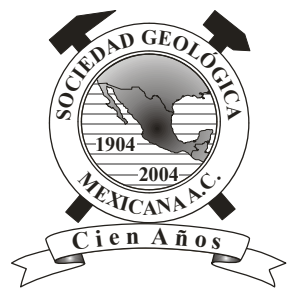

\title{
Contribución al alcance estratigráfico de la Formación Agueguexquite (Mioceno), Veracruz, México
}

\author{
Gabriela Pineda-Salgado ${ }^{1}$, Peter Schaaf ${ }^{2}$, Marcelo Aguilar-Piña ${ }^{3}$, Gabriela Solís-Pichardo $^{4}$, \\ Francisco J. Vega ${ }^{4, *}$ \\ ${ }^{1}$ Facultad de Ciencias Naturales y Museo, Universidad Nacional de La Plata, La Plata Buenos Aires 1900, Argentina. \\ ${ }^{2}$ Instituto de Geofísica, Universidad Nacional Autónoma de México, Ciudad Universitaria, Coyoacán, CDMX, 04510, México. \\ ${ }^{3}$ Instituto Mexicano del Petróleo, Gustavo A. Madero, México, CDMX, 07730, México. \\ ${ }^{4}$ Instituto de Geología, Universidad Nacional Autónoma de México, Ciudad Universitaria, Coyoacán, CDMX, 04510, México. \\ *vegver@unam.mx
}

\section{Resumen}

La Formación Agueguexquite aflora en el sureste de México, en el sur de Veracruz y norte de Chiapas. Su edad aún no ha sido determinada formalmente, estudios previos han considerado su depósito entre el Mioceno y el Plioceno. Moluscos y muestras de roca de tres localidades de la Formación Agueguexquite en el estado de Veracruz, fueron recolectados a fin de establecer el alcance estratigráfico de la formación por medio de edades relativas utilizando foraminíferos bentónicos y nanoplancton calcáreo, así como estratigrafía isotópica $\left(\mathrm{Sr}^{87} / \mathrm{Sr}^{86}\right)$. La datación con foraminíferos bentónicos mostró un alcance estratigráfico amplio (Mioceno-Plioceno). La nanofauna calcárea muestra una edad de depósito del Mioceno medio-tardío, que fue corroborada con los resultados de la estratigrafía isotópica con ( $\mathrm{Sr}^{87} /$ $\left.\mathrm{Sr}^{86}\right)$. Con base a los resultados obtenidos se sugiere que la Formación Agueguexquite fue depositada entre el Mioceno medio y tardío.

Palabras clave: Formación Agueguexquite, Mioceno, estratigrafía, Veracruz, México.

\begin{abstract}
The Agueguexquite Formation crops out in southeastern Mexico, in southern Veracruz and northern Chiapas; its age has not been formally determined, but previous studies consider that it was deposited between the Miocene and Pliocene. Mollusks and rock samples from three localities of the Agueguexquite Formation in Veracruz were collected in order to define the stratigraphic range of this formation by means of relative ages using benthic forams and calcareous nannoplankton, as well as isotopic stratigraphy $\left.{ }^{87} \mathrm{Sr}{ }^{86} \mathrm{Sr}\right)$. Dating with benthic forams indicates a broad stratigraphic range (Miocene-Pliocene); calcareous nannoplankton yielded a middle-late Miocene age, which is supported by the results of isotopic stratigraphy $\left({ }^{87} \mathrm{Sr}{ }^{86} \mathrm{Sr}\right)$. Based on our stratigraphic results, it is suggested that the Agueguexquite Formation was deposited between the middle and late Miocene.
\end{abstract}

Keywords: Agueguexquite Formation, Miocene, stratigraphy, Veracruz, Mexico.

\section{Introducción}

La Formación Agueguexquite aflora en el sureste de México, en la Cuenca Salina del Istmo. Las principales localidades se encuentran a lo largo del sur del estado de
Veracruz (Figura 1.1), en Santa Rosa y Santa Lucrecia, hasta Zuzulum, en el estado de Chiapas. Predominan secuencias terrígenas de origen litoral (Ayala-Garduño, 2003). La Formación Agueguexquite fue definida por Thalman (1934), quien definió la localidad tipo en una serie de afloramientos 
en el cruce del camino de Puerto México a Santa Rosa, cerca del arroyo Agueguexquite, Veracruz. En su base presenta un alto contenido de moluscos y foraminíferos en excelente estado de preservación (Perrilliat, 1960; Graham, 1994). Se encuentra en discordancia sobre la Formación Paraje Solo del Mioceno medio; discordantemente está cubierta por la Formación Cedral del Mioceno tardío (Castillo-Tejero, 1955; Perrilliat, 1960; López-Ramos, 1979). Según CastilloTejero (1955), la formación se divide en tres partes; la parte inferior está compuesta por arenisca color café y arcilla muy arenosa, con abundantes foraminíferos; la parte media se compone de una arenisca compactada de grano fino a medio, ligeramente fosilífera y la superior está formada por arcilla lignítica de color azul grisáceo, con fósiles preservados como moldes.

La edad de la Formación Agueguexquite aún no ha sido determinada formalmente, ya que algunos autores consideraron que pertenece al Mioceno medio o al Mioceno tardío. Thalman (1934) la consideró como el límite entre el Mioceno Inferior y el Mioceno Medio, posteriormente fue ubicada en el Mioceno Superior por Castillo-Tejero (1955). Perrilliat (1963) determinó que la edad de los afloramientos de la Formación Agueguexquite cercanos a Coatzacoalcos es Mioceno medio, por la presencia de la especie Anadara (Diluvarca) strebla Gardner, 1947; esta especie es un fósil característico del Mioceno medio que sólo se había encontrado en la Formación Shoal River, Florida. La misma autora determinó que esta unidad podía correlacionarse con depósitos miocénicos del sureste de Estados Unidos, el Caribe, América Central y Sudamérica (Perrilliat, 1972). Sansores y Flores-Covarrubias (1972) consideraron que la unidad pertenecía a la base del Mioceno Superior. Los estudios sobre ostrácodos de Bold (1977) determinaron una edad pliocénica. Herrera-Anduaga (1979) determinó que el momento de depósito ocurrió durante el Mioceno-Plioceno, a partir de la distribución temporal de algunas especies de briozoarios. Akers (1979), a través de un trabajo estratigráfico en la Formación Paraje Solo y Concepción, concluyó que la Formación Agueguexquite debía ser considerada perteneciente al Plioceno Medio, ya que de acuerdo a su interpretación, la fauna marina de esta unidad era contemporánea a la que se presenta en la Formación Paraje Solo. Recientemente, Perrilliat y FloresGuerrero (2011) ubicaron a la Formación Agueguexquite como Plioceno Inferior.

Dada la problemática planteada, el objetivo de este trabajo es establecer la edad de la Formación Agueguexquite en tres afloramientos, a través de dataciones relativas con foraminíferos bentónicos y nanoplancton calcáreo, y con estratigrafía isotópica de estroncio $\left({ }^{87} \mathrm{Sr} /{ }^{86} \mathrm{Sr}\right)$.

El contenido paleontológico ha sido estudiado ampliamente a partir de los trabajos de Perrilliat (1960; $1963 ; 1972 ; 1973 ; 1974 a ; 1974 b ; 1976 ; 1977 ; 1984)$ sobre la fauna de gasterópodos y bivalvos. Bold (1977) estudió la fauna de ostrácodos y Sansores y Flores-Covarrubias (1972) estudiaron los foraminíferos presentes en la formación
(Herrera-Anduaga, 1979). Herrera-Anduaga (1979; 1983) realizó estudios sobre la fauna de briozoarios. Vega et al. (2009) reportan dos especies de crustáceos decápodos. Perrilliat y Flores-Guerrero (2011) reportaron la fauna de moluscos en las localidades de El Tepache, Coatzacoalcos y Nuevo Teapa, entre las que se incluyeron nuevos registros. Finalmente, Pineda-Salgado et al. (2011) realizaron un estudio en el que se reconocieron trazas de depredación en gasterópodos y bivalvos de las localidades previamente estudiadas por Perrilliat y Flores-Guerrero (2011). Los datos preliminares de ese estudio aportaron información que contribuye a la comprensión de los eventos de extinción que ocurrieron en el Neógeno tardío en el Atlántico.

Se ha considerado que la Formación Agueguexquite representa una fase transgresiva, (Castillo-Tejero, 1955), debido a que el ambiente de la Formación Paraje Solo es salobre; el contacto entre ésta y la Formación Agueguexquite es evidente por un conglomerado basal y la presencia de fósiles marinos.

\section{2. Área de estudio}

Se visitaron tres localidades en el estado de Veracruz (Figura 1.1): I-Nuevo Teapa (I-NT), en un corte sobre la Carretera Federal 180, cerca del poblado de Nuevo Teapa, al este de Nanchital; II-Córdoba-Minatitlán (II-CM), en la intersección de las carreteras Córdoba-Minatitlán y Coatzacoalcos-Cárdenas, al sureste de Nuevo Teapa y al norte de Ixtlahuatlán del Sur (Figura 1.2). En la localidad I predomina la arenisca café y rojiza, mientras que en la localidad II aflora lutita de color negro. La tercera localidad se denominó III-Río Coatzacoalcos (III-RC), al margen del río con el mismo nombre, en el municipio de Jesús Carranza, cerca del poblado El Tepache, al sur de Ojo de Agua y al norte de López Mateos, a 29.7 km de la cabecera municipal (Figura 1.3). En esta localidad predomina arenisca, lutita y limolita de color gris claro. Cerca de la localidad la litología que predomina son la lutita, la toba riolítica y la arenisca con limolita. Aunque en las tres localidades la presencia de moluscos fósiles es frecuente (Figura 2), en este trabajo nos enfocaremos en los datos proporcionados por microfósiles y datación isotópica. Los ejemplares de moluscos están depositados en la colección del Museo de Paleontología de la Facultad de Ciencias, Universidad Nacional Autónoma de México, bajo el acrónimo FCMP.

\section{Método}

\subsection{Datación relativa}

A partir de muestras de roca procedentes de la localidad III-RC, se obtuvo una pequeña muestra de foraminíferos (RC0211), que fueron determinados hasta nivel de género y especie. Se utilizaron dos muestras de roca (Agx1 y 

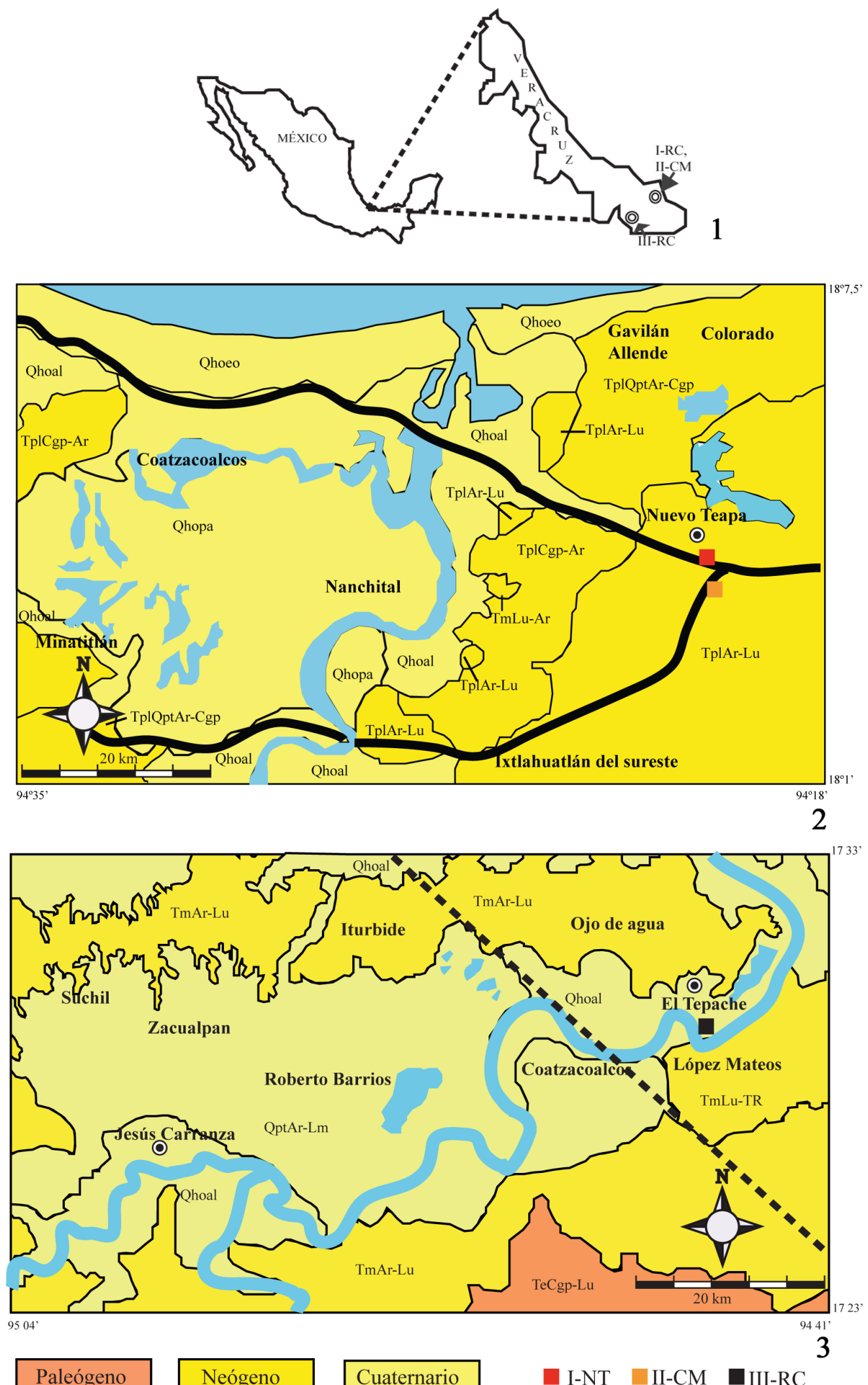

Figura 1. Área de estudio. 1, Ubicación de las tres localidades estudiadas de la Formación Agueguexquite (NT, CM y RC). 2, Localidades I-NT en rojo y II-CM en verde (Modificada de Carta Geológica-Minera de Minatitlán). 3, Localidad III-RC en rojo (Modificada de Carta Geológica-Minera Coatzacoalcos). Acotaciones: Qhoal, aluvión; Qhopa, palustre; Qhoeo, élico; QptAr-Lm, arena-limo; TeCgp-Lu, conglomerado oligomíctico-lutita; TplQptAr-Cgp, conglomerado oligomíctico-arenisca; TplCgp-Ar, conglomerado polimíctico-arenisca; TmAr-Lu y TplAr-Lu, arena-lutita; TmLu-TR, lutita-toba riolítica; azul cuerpo de agua; línea punteada, Falla Río Solosuchil, de acuerdo a las cartas antes citadas. 


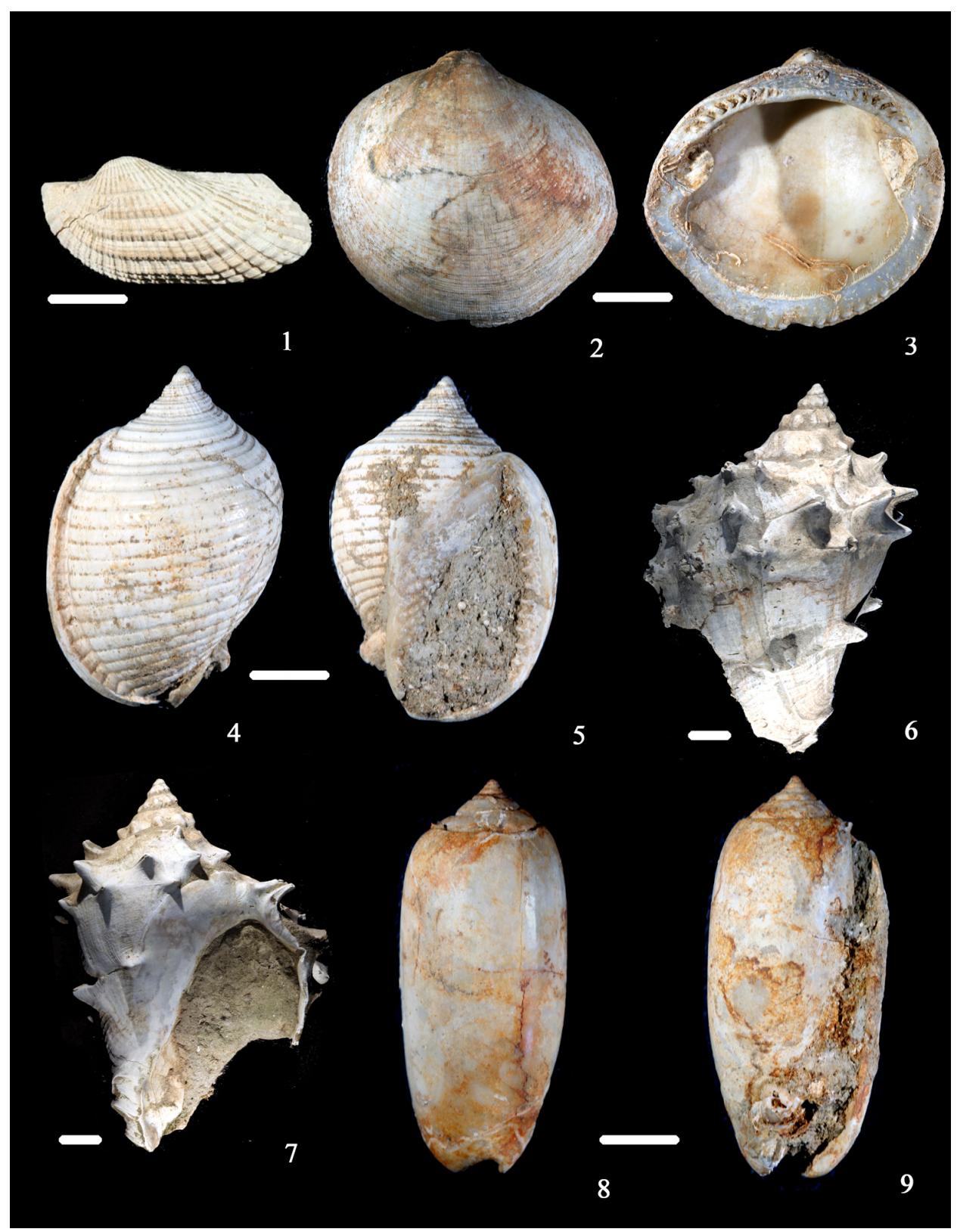

Figura 2. Moluscos del Mioceno de la Formación Agueguexquite. 1, Anadara (Rasia) lienosa Say, 1830, ejemplar FCMP-1145, localidad II-CM, Messiniano, Mioceno. 2, 3, Glycymeris (Glycymeris) sp., ejemplar FCMP-1146, localidad I-NT, Tortoniano, Mioceno. 4, 5, Phalium alligator Petuch, 1991, ejemplar FCMP-1147, localidad I-NT, Tortoniano, Mioceno. 6, 7, Melongena consors? Sowerby, 1850, ejemplar FCMP-1148, localidad III-R, Langhiano, Mioceno. 8-9, Oliva lioides Dall, 1903, ejemplar FCMP-1149, localidad I-NT, Tortoniano, Mioceno.

Agx2) con el fin de encontrar y determinar de nanoplancton calcáreo, que permitiera establecer una posible edad para el afloramiento.

\subsection{Estratigrafía Isotópica}

Las muestras para la estratigrafía isotópica se obtuvieron a partir de conchas de moluscos (bivalvos y gasterópodos) de cada localidad (I-NT, II-CM y III-RC), las cuales fueron molidas y etiquetadas para posteriormente ser fechadas (datación relativa en comparación con la curva del agua del mar) con la técnica ${ }^{87} \mathrm{Sr} r{ }^{86} \mathrm{Sr}$ en el Laboratorio Universitario de Geoquímica Isotópica (LUGIS) del Instituto de Geofísica, Universidad Nacional Autónoma de México. Los análisis isotópicos se realizaron con un espectrómetro de masas con fuente iónica térmica marca THERMO SCIENTIFIC modelo TRITON PLUS. El espectrómetro cuenta con 9 colectores Faraday ajustables y todas las mediciones se hicieron de manera estática. Las muestras de estroncio ( $\mathrm{Sr}$ ) se cargaron como cloruros y se midieron como iones metálicos. Se analizaron 60 relaciones isotópicas $(\mathrm{n})$ para Sr. Los errores $\left(1 \mathrm{sd}= \pm 1 \sigma_{\text {abs }} ; 2 \mathrm{SE}(\mathrm{M})=2 \sigma_{\text {abs }}\right.$ /raíz n $)$ 
durante la medición se refieren en los últimos dos dígitos. Todas las relaciones isotópicas de $\mathrm{Sr}$ se corrigieron por fraccionamiento de masas vía normalización a ${ }^{86} \mathrm{Sr} r{ }^{87} \mathrm{Sr}=$ 0.1194. Los valores del LUGIS para el estándar NBS 987 $(\mathrm{Sr}):{ }^{87} \mathrm{Sr} /{ }^{86} \mathrm{Sr}=0.710239 \pm 12\left( \pm 1 \sigma_{\text {abs }}, \mathrm{n}=18\right)($ Tabla 1$)$. Las edades relativas se obtuvieron con el programa LOWESS (versión 3) de McArthur et al. (2001).

\section{Resultados}

\subsection{Datación relativa}

En la muestra RC0211 se determinaron únicamente foraminíferos bentónicos de las especies Amphistegina lessonii (d'Orbigny, 1826) y Lenticulina americana (Cushman, 1918) (Figura 3). Estas especies han sido referidas para ambientes marinos cálidos y someros, con una profundidad menor a $20 \mathrm{~m}$ (Thomas, 2005). El alcance estratigráfico de las dos especies es muy extenso (MiocenoPleistoceno), sin embargo, Sansores y Covarrubias (1972) mencionan que $A$. lessonii había sido referida para las formaciones Paraje Solo (Pleistoceno), Agueguexquite (Pleistoceno), Filisola (Plioceno) y Encanto (Mioceno Superior). Por otra parte, McLean (1951) mencionó que Lenticulina americana es común para la Formación Jackson Bluff del Mioceno de Florida. Los foraminíferos determinados indican un ambiente marino de escasa profundidad y cálido que cronoestratigráficamente puede ir del Mioceno al Pleistoceno.

Las zonaciones de nanoplancton calcáreo que se utilizan en la actualidad para el Cenozoico, fueron realizadas por Martini (1971), quien dividió al periodo en 46 zonas y por Okada y Bukry (1980) que dividieron al Cenozoico en 34 zonas y 46 subzonas, con esto es posible realizar correlaciones a nivel mundial, lo que permite colocar las dataciones con nanofósiles en un plano importante de la estratigrafía (Lancis-Sáez, 1998). En este trabajo, se utilizó la zonación de Martini (1971).

Para nanoplancton calcáreo, en la muestra Agx1 se determinaron las especies Sphenolithus abies Deflandre en Deflandre y Fert, 1954 “A”, S. abies "B”, S. moriformis (Brönnimann y Stradner, 1960), Discoaster neohamatus (Bukry y Bramlette, 1969), D. pentaradiatus (Tan, 1927), D. surculus (Martini y Bramlette, 1963), Discoaster sp. (6 brazos), Helicosphaera paleocarteri (Theodoridis, 1984), H. kamptneri (Hay y Mohler en Hay et al., 1967), H. sellii (Bukry y Bramlette, 1969), Cocolithus pelagicus (Wallich, 1877), Calcidiscus leptoporus (Murray y Blackman, 1898), C. macintyrei (Bukry y Bramlette, 1969), Braarudosphaera bigelowii (Gran y Braarud, 1935), Umbilicosphaera sibogae (Weber - van Bosse, 1901) y U. sibogae foliosa (Kamptner, 1963), considerando como especies retrabajadas a Dictyococcites bisectus (C), Predicosphaera cretacea (Arkhangelsky, 1912), Eiffellithus turrisseiffelii (Deflandre en Deflandre y Fert, 1954), Nannoconus sp., Pontosphaera multipora (Kamptner, 1948) y Pontosphaera sp. Las especies S. moriformis, D. neohamatus, D. pentaradiatus, D. surculus (Figura 4) permiten ubicar la muestra en la base del Mioceno Superior.

En la muestra Agx2 se determinaron las especies Sphenolithus abies "A", S. abies "B", S. moriformis, Discoaster neohamatus, D. pentaradiatus, D. surculus, D. challengeri (Bramlette y Riedel, 1954), D. bollii (Martini y Bramlette, 1963), D. brouweri (Tan, 1927), Discoaster sp. (6 brazos), Discoaster sp. (5 brazos), Helicosphaera paleocarteri, H. sellii, Coccolithus pelagicus (Wallich 1877), Calcidiscus leptoporus, C. macintyrei, Umbilicosphaera sibogae, U. sibogae foliosa y Scyphosphaera pulcherrima (Deflandre, 1942); con las especies retrabajadas: Cyclicargolithus floridanus (Roth y Hay, en Hay et al., 1967) "B”, Triquetrorhabdulus carinatus Martini, 1965, Blackites tenuis (Bramlette y Sullivan, 1961), Predicosphaera cretacea, Eiffellithus turrisseiffelii, Micula staurophora (Gardet, 1955).

La edad de la microfauna se ubicó a partir de las especies Sphenolithus abies "A", Sphenolithus abies "B", Discoaster challengeri, Discoaster bollii y Discoaster brouweri (Figura 4) como parte superior del Mioceno Medio.

Finalmente, a partir de la nanofauna calcárea y con base a la zonación de Martini (1971), (biozonas NN7, NN8 y NN9) se estableció que la Formación Agueguexquite se depositó entre el Mioceno medio y el Mioceno tardío.

\subsection{Estratigrafía isotópica}

Los blancos analíticos obtenidos en el tiempo de los análisis de las muestras de este trabajo resultaron: 0.4 ng Sr. La Tabla 1 muestra los valores obtenidos con el espectrómetro de masas TRITON PLUS.

Con base en las edades obtenidas en millones de años y a partir del cuadro cronoestratigráfico de 2015 de la Comisión Internacional de Estratigrafía (Cohen et al., 2013; updated), se establecen las edades en las que se depositó la Formación Agueguexquite. La localidad III-RC (muestra RC2602II) corresponde al Burdigaliano-Langhiano (15.99 $+0.78 /-1.31 \mathrm{Ma}$ ) (Mioceno temprano-medio), mientras que las localidades I-NT (muestra NT2502II) y II-CM (muestra CM2502II), corresponden al Tortoniano (8.87 $+1.77 /-2.26 \mathrm{Ma})$ y Messiniano (6.87 "+ $2.16 /-0.88$ $\mathrm{Ma}$ ), respectivamente (Mioceno tardío). Por lo anterior se asume que las localidades estudiadas de la Formación Agueguexquite corresponden del Mioceno Medio al Mioceno Superior (Burdigaliano-Messiniano) (Figura 5).

\section{Discusión}

Es importante señalar que en las cartas GeológicoMinera de Minatitlán E15-7 (SGM, 2000), Coatzacoalcos E15-1-4 (SGM, 2004) y Veracruz E14-3 (SGM, 2002) no figura la Formación Agueguexquite, considerando entonces 
Tabla 1. Resultados de la datación absoluta, en donde $\mathrm{n}$ es el número de relaciones medidas por corrida; 1sd corresponde a una desviación estándar; $2 \mathrm{SE}(\mathrm{M})=2 \mathrm{sd} / \sqrt{ } \mathrm{n}$.

\begin{tabular}{|c|c|c|c|c|c|c|c|c|c|}
\hline \# & Muestra & Código & ${ }^{87} \mathrm{Sr} /{ }^{86} \mathrm{Sr}$ & $1 \mathrm{sd}^{*}$ & $2 \mathrm{SE}(\mathrm{M})$ & $\mathrm{n}$ & Tipo & $\mathrm{Ma}$ & $\pm \mathrm{Ma}$ \\
\hline & Std SRM 987 & SrT055 & 0.710240 & 37 & 5 & 58 & estándar & & \\
\hline 1 & NT 2502 II & 4684 VV CR & 0.708916 & 37 & 5 & 58 & concha & 8.87 & $"+1.77 /-2.26$ \\
\hline 2 & RC 2602 II & 4685 VV CR & 0.708745 & 37 & 5 & 58 & concha & 15.99 & $"+0.78 /-1.31$ \\
\hline 3 & CM 2502 II & 4686 VV CR & 0.708952 & 32 & 4 & 58 & concha & 6.87 & $"+2.16 /-0.88$ \\
\hline
\end{tabular}

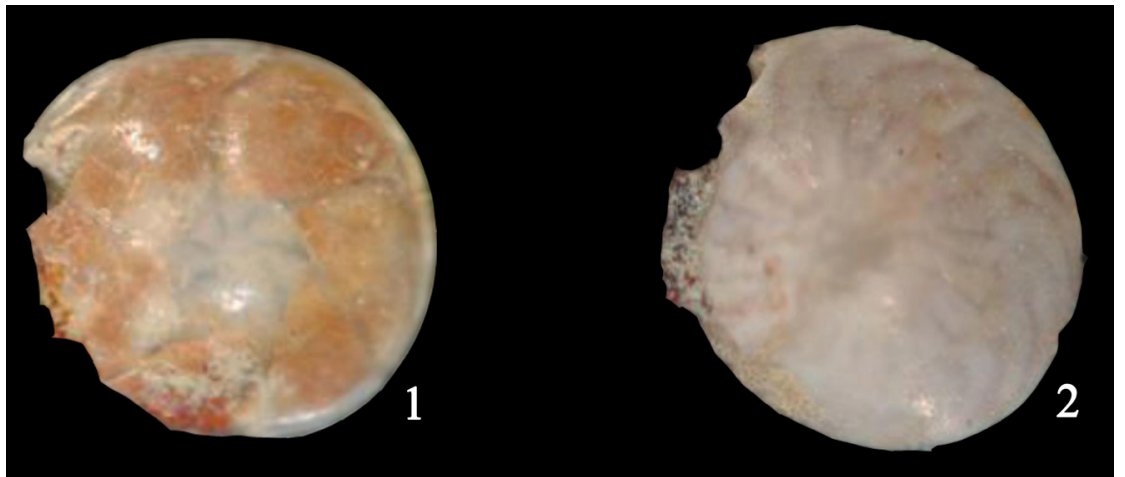

Figura 3. Foraminíferos bentónicos de la localidad III-RC. 1, Lenticulina americana x 32. 2, Amphistegina lessonii x 32.
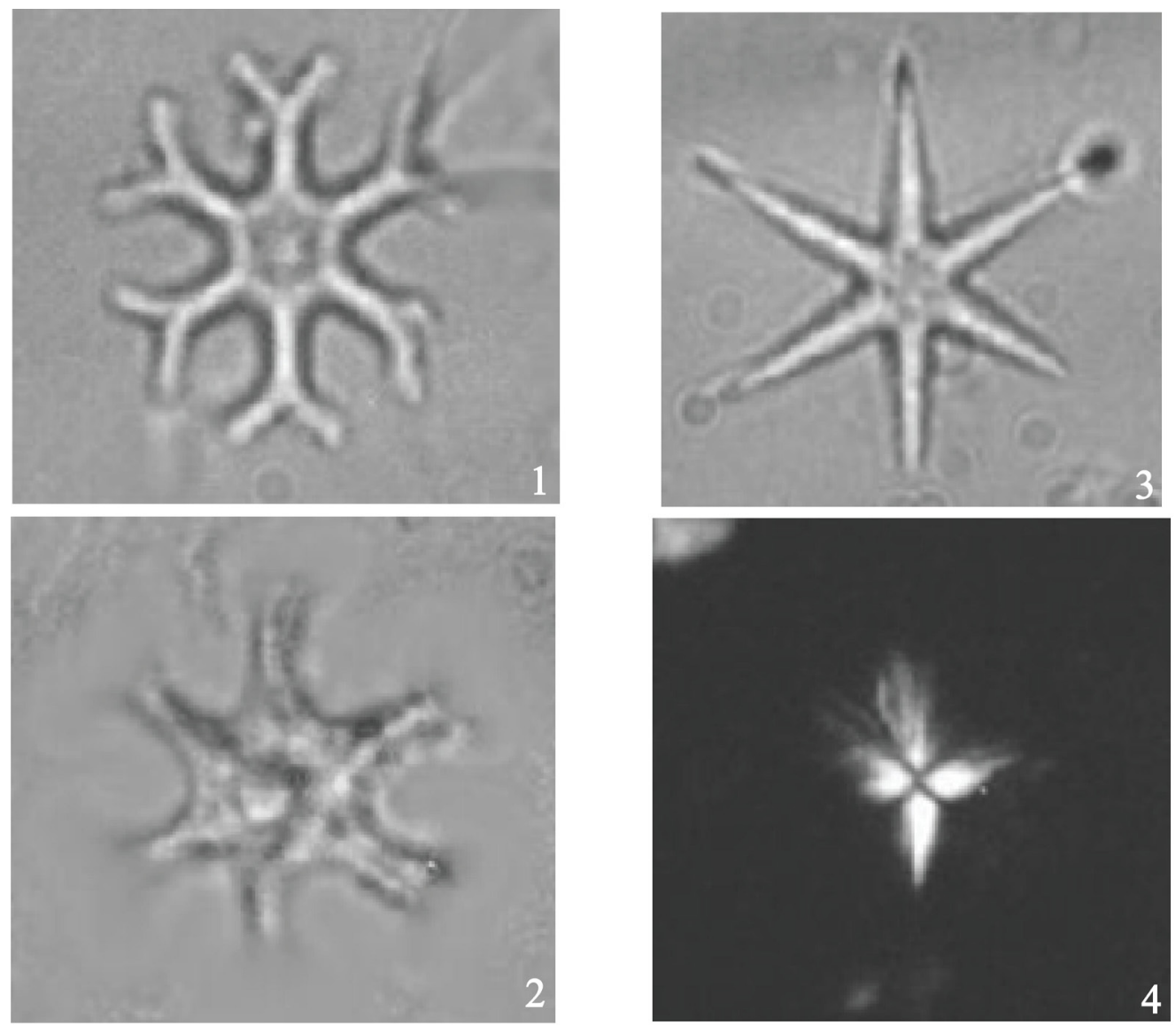

Figura 4. Nanoplancton calcáreo de la localidad III-RC. 1, Discoaster challengeri x 1600, luz natural. 2, D. brouweri x 1600, luz natural. 3 , D. bollii x 1600, luz natural. 4, Sphenolithus abies x 1600 luz polarizada. 


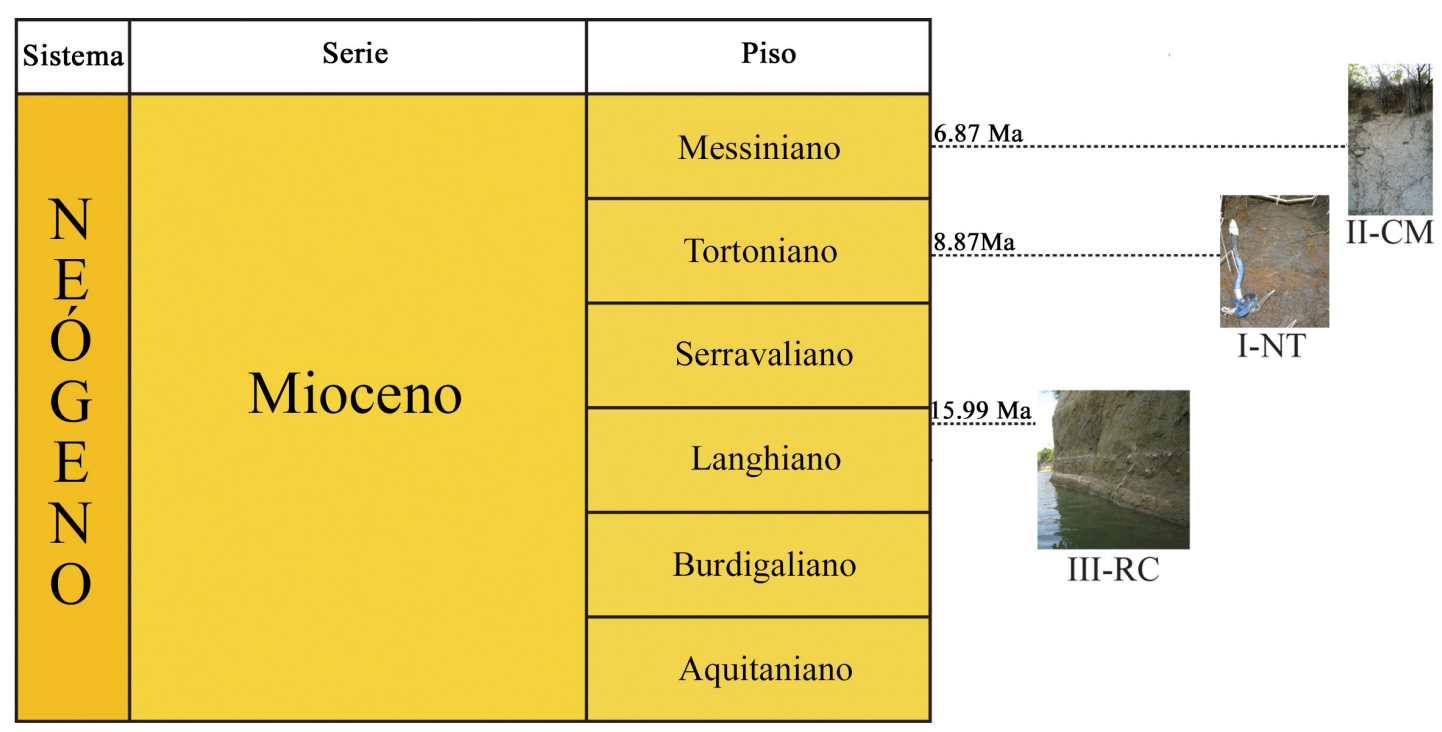

Figura 5. Edad de las localidades estudiadas, I-NT, II-CM y III-RC, obtenidas a partir de estratigrafía isotópica con estroncio.

que los afloramientos estudiados pertenecen a la Formación Concepción; sin embargo, ésta pertenece a la Cuenca de Veracruz y sus afloramientos se caracterizan por una sucesión de arenisca y lutita con concreciones de areniscas cementadas con material calcáreo. Por otra parte, en el léxico estratigráfico del Servicio Geológico Mexicano se incluye a la Formación Agueguexquite en la Cuenca Salina del Istmo. Sáenz-Pita y López-Palomino (2012) consideran que su edad es Pliocénica, considerando los trabajos de Akers (1979) y Waller (2011), este último en su trabajo sistemático sólo cita la Formación Agueguexquite por la presencia del pectínido Nodipecten collirensis floridensis Tucker y Wilson, 1933 y menciona que la edad pliocénica fue correlacionada por Akers (1974) con los depósitos de Pinecrest, Florida.

Con las muestras de roca recolectadas en la localidad III-RC, se contó con elementos suficientes para determinar un intervalo de edad de la Formación Agueguexquite, a través de nanoplancton.

Considerando los registros de la Tabla 2, se puede observar que las edades que han asignado previamente para la Formación Agueguexquite van del Mioceno temprano al Plioceno medio y, a pesar del desacuerdo que aún existe, se puede ver que los resultados obtenidos en este trabajo coinciden con la datación realizada por Gibson (1936), Castillo-Tejero (1955), Romen (1955), Contreras (1956), PEMEX (1956), Perrilliat (1963) y Sansores y Flores-Covarrubias (1972); también coincide con la edad propuesta por Herrera-Anduaga (1979). Por otra parte, existen otros reportes como los de Bold (1978), Akers (1972; 1979), Perrilliat y Flores-Guerrero (2011) que ubican a la formación en el Plioceno temprano y Plioceno medio.

La edad obtenida para este trabajo con foraminíferos bentónicos muestra un intervalo de edad muy amplio, incluso la utilidad bioestratigráfica de estos organismos se ha limitado debido a que pocas formas presentan rangos estrechos de distribución en el tiempo. A pesar de ello, se han utilizado en algunas ocasiones para realizar correlaciones a nivel regional y mundial (Tchegliakova et al., 1997), inclusive son utilizados para trabajos estratigráficos en el norte de México, pero su utilidad se restringe a la determinación de condiciones paleoambientales. Considerando su bajo potencial estratigráfico y que el número de muestras analizadas no era significativo en diversidad ni en abundancia, sólo se consideró la información paleoambiental que brindaron.

A diferencia de éstos, con el nanoplancton calcáreo se obtuvo un rango de edad más cerrado en el tiempo geológico. Retomando las edades que se propusieron previamente, es posible observar el uso de foraminíferos y nanoplancton calcáreo al igual que otros organismos como briozoarios y moluscos. A continuación se discute la utilidad de estos organismos en la bioestratigrafía. Los foraminíferos y el nanoplancton son ampliamente usados para la determinación de edades relativas, estos organismos fueron utilizados por Bold (1978) y Akers (1972; 1979). El caso que se discutirá es el trabajo de Akers (1979), en el cual ubica a la Formación Agueguexquite en la zona No. 20 de Blow (1969) y Berggren (1969), por la presencia de Sphenolithus abies, con una distribución temporal que abarca del Mioceno tardío al Plioceno medio; destacando el límite de la zona con la extinción de esta especie en el Plioceno medio. Akers (1979) considera que la Formación Agueguexquite pertenece al Plioceno medio por la presencia de las siguientes especies: Pseudoemiliania lacunosa Kamptner, 1963, Globigerina bulloides apertura Cushman, 1918 y Sphaeroidinella dehiscens (Parker et al., 1865) forma inmatura; esta última característica del Plioceno medio y realiza una correlación con las formaciones Yorktown (Virginia y Carolina del Norte) y Jackson Bluff (Florida); 
Tabla 2. Edades establecidas para la formación Agueguexquite por métodos relativos.

\begin{tabular}{ll}
\hline \multicolumn{1}{c}{ Autor (año) } & \multicolumn{1}{c}{ Edad } \\
\hline Thalman (1934) & Mioceno temprano-medio \\
Gibson (1936)* & Mioceno medio \\
Castillo-Tejero (1955) & Mioceno tardío \\
Romen (1955)* & Mioceno tardío \\
Contreras (1956)* & Mioceno tardío \\
PEMEX (1956)* & Mioceno medio \\
Perrilliat (1963) & Mioceno medio \\
Sansores y Flores- & Mioceno medio \\
Covarrubias (1970) & Plioceno temprano \\
Akers (1972) & Plioceno \\
Van Den Bold (1977) & Plioceno medio \\
Akers (1979) & Mioceno tardío-Plioceno \\
Herrera-Anduaga (1979) & temprano \\
Perrilliat-Montoya y Flores- & Plioceno temprano \\
Guerrero (2011) & Mioceno medio-tardío \\
Este trabajo & \\
\hline
\end{tabular}

sin embargo, actualmente se considera que la Formación Yorktown pertenece al Mioceno tardío-Plioceno temprano (Hazel, 1971; Kier, 1972), mientras que la Formación Jackson Bluff es considerada parte de depósitos de Plioceno temprano (Means, 2010).

Finalmente, Akers (1979) señaló que la nanoflora calcárea de la Formación Agueguexquite es más joven que la de sus equivalentes en Estados Unidos. Esta observación remite a su trabajo (Akers, 1972), en donde determina que la unidad se ubica estratigráficamente en el Plioceno temprano; este trabajo fue retomado por Perrilliat y Flores-Guerrero (2011), pero su estudio no brinda una justificación de sus resultados que soporte los trabajos de Akers (1972). También es importante destacar que en el trabajo de Herrera-Anduaga (1979), se asigna una edad en el límite Mioceno-Plioceno, a partir del registro de briozoarios. Herrera-Anduaga (1979) considera el registro estratigráfico de Membraniporidra oecioporosa Canu y Bassler, 1920, que aparece en el Eoceno tardío y se extingue en el Mioceno tardío y los registros de Cellaria bassleri Hastings, 1947 y Antropora typica (Canu y Bassler, 1928), que surgen en el Plioceno y están representadas actualmente. Al contar con la presencia de estas tres especies en el mismo conjunto faunístico, señala un intervalo de tiempo restringido, ya que a partir de su distribución temporal, sólo pudieron coexistir durante un intervalo de tiempo muy corto. Con este aporte, la misma autora señala que los briozoarios pueden ser indicadores de edad, reconociendo que las edades obtenidas no son tan precisas como la que se pueden obtener con foraminíferos. A pesar de que algunos autores consideran que los briozoarios no deben emplearse como fósiles índice debido a su amplio alcance estratigráfico, debe tomarse en cuenta el aporte de Herrera-Anduaga (1979), ya que sólo utiliza el intervalo de tiempo en el que estas tres especies coinciden, utilizando una Zona de Oppel. Otro factor que favorece la edad obtenida con los briozoarios es que, las formaciones que Akers (1979) considera como contemporáneas, Formación Yorktown (Mioceno Superior) y Formación Jackson Bluff (Plioceno temprano), tienen un rango de edad similar para la Formación Agueguexquite.

En este trabajo se establece que la Formación Agueguexquite se depositó durante el Mioceno mediotardío a partir de las especies de nanoplancton calcáreo de la Tabla 3; pero al considerar su alcance estratigráfico se puede observar la variación que hay en el mismo, ya que se encontraron especies como Discoaster neohamatus, que se restringe al Mioceno tardío (en estratigrafía es una especie característica de la zona NN9), o especies como Discoaster brouweri cuyo alcance estratigráfico abarca del Mioceno medio al Plioceno tardío.

En este punto, es posible visualizar el problema que implica la determinación de la edad de una formación, sin embargo la coincidencia entre los ejemplares estudiados en este trabajo, brindan un rango de edad que coincide con el de otros autores mencionados con anterioridad, a pesar de que las referencias bibliográficas que existen apoyando edades miocénicas y pliocénicas para la formación son muy extensas en ambas partes. Si no se contara con una datación absoluta, podría proponerse un consenso de edad, definiendo a la Formación Agueguexquite como un depósito del Mioceno tardío; esta propuesta podría soportarse en el hecho de que todas las especies de la Tabla 3 se sobrelapan en esta edad. Con esta propuesta temporal se busca contrarrestar los alcances y las limitaciones que brindan los diferentes organismos que han sido utilizados como fósiles índice para asignarle una edad a la Formación Agueguexquite.

Por otra parte, los resultados que se obtuvieron con el sistema isotópico con estroncio ( $\mathrm{Sr}$ ), confirman la edad que se estableció a través de la nanofauna y muestra que en ausencia de estos resultados, el consenso de edad no hubiera sido erróneo. Al comparar la validez de los métodos que se utilizaron en este trabajo, es importante considerar que para la datación relativa con foraminíferos y nanoplancton calcáreo sólo se consideró la localidad III-RC, mientras que la estratigrafía isotópica consideró las localidades I-NT, IICM y III-RC. La edad de cada una de estas localidades nos permite conocer el orden en que se depositaron y con ayuda de la litología es posible conocer el ambiente del mismo.

La localidad III-RC es la más antigua, ya que se depositó durante el Mioceno temprano-medio, esta localidad se caracteriza por la presencia de lutita y arenisca con limo. La arenisca y el limo son comunes en ambientes marinos someros, coincidiendo con los organismos presentes en el área (moluscos, corales y briozoarios). 
Tabla 3. Alcance estratigráfico de las especies que fueron utilizadas en este trabajo para establecer la edad relativa de la Formación Agueguexquite. Rangos de Nannotax, Young et al. (2013).

\begin{tabular}{|c|c|c|c|c|c|}
\hline \multirow{2}{*}{ Especies } & \multicolumn{3}{|c|}{ Mioceno } & \multicolumn{2}{|c|}{ Plioceno } \\
\hline & temprano & medio & tardío & temprano & tardío \\
\hline \multicolumn{6}{|l|}{ Sphenolithus abies } \\
\hline \multicolumn{6}{|l|}{ Discoaster neohamatus } \\
\hline \multicolumn{6}{|l|}{ Discoaster pentaradiatus } \\
\hline \multicolumn{6}{|l|}{ Discoaster surculus } \\
\hline \multicolumn{6}{|l|}{ Discoaster challengeri } \\
\hline \multicolumn{6}{|l|}{ Discoaster bollii } \\
\hline Discoaster brouweri & & & & & \\
\hline
\end{tabular}

Los depósitos de las localidades I-NT y II-CM muestran una trasgresión marina. La presencia de arenisca y lutita con tonalidades que van de café a negro, sugiere que el contenido de materia orgánica es elevado en un medio anóxico, bajo este supuesto puede explicarse la ausencia de organismos en la localidad I-NT.

En la localidad II-CM la tonalidad del sedimento era café lo cual indica un ambiente oxigenado con un contenido de materia orgánica menor, si se comparara con la localidad I-NT. La presencia de algunos moluscos y corales refuerza el hecho de que la localidad II-CM pertenecía a un ambiente marino somero. Aunque el depósito de esta localidad es ligeramente más reciente o contemporáneo a la localidad I-NT, es evidente que ambas reflejan cambios en el nivel del mar. En la localidad I-NT se ha reportado la presencia de capas rojas y grises, estas intercalaciones de estratos podrían relacionarse con cambios en el nivel del mar. Se ha observado que entre las capas hay superficies de erosión. Las capas grises pueden compararse con ambientes profundos de las costas del Pacífico de Panamá (Herbert, com. pers.).

Con estas interpretaciones es evidente que la estratigrafía isotópica y la datación relativa no han sido excluyentes para poder interpretar y conocer la historia de depósito de la formación. Es importante señalar que ambos métodos muestran limitaciones al indicar la edad de algún conjunto de rocas. Una de las deficiencias con estratigrafía isotópica que se presenta en este trabajo es que los carbonatos biogénicos no contienen una fracción detrítica por ser secretados directamente del agua marina por los organismos, generando un escaso contenido de $\mathrm{Rb}$, por lo que suele emplearse una calibración de la evolución de las relaciones isotópicas de ${ }^{87} \mathrm{Sr} /{ }^{86} \mathrm{Sr}$ que termina siendo una herramienta indirecta para el fechamiento (LUGIS, 2011).

\section{Conclusiones}

Se estableció una edad para el depósito de la Formación Agueguexquite a través de bioestratigrafía con nanoplancton calcáreo y estratigrafía isotópica con estroncio $\left({ }^{87} \mathrm{Sr} /{ }^{86} \mathrm{Sr}\right)$, ambos métodos mostraron un rango temporal equivalente; si bien ambas dataciones permitieron establecer una edad cercana, con la estratigrafía isotópica fue posible conocer la edad de depósito para cada localidad de estudio.

Con el conocimiento de la edad de la formación, es posible continuar con trabajos relacionados con los eventos de extinción regional masiva que ocurrieron en la costa oeste del Océano Atlántico durante el Neógeno tardío, si bien los afloramientos de las costas de Estados Unidos están ampliamente estudiados y se tiene documentado parte del impacto de la extinción espacial y temporalmente, es importante considerar conjuntos de moluscos fósiles de la Formación Agueguexquite para conocer si existen similitudes espaciales, temporales y de impacto entre especies y familias registradas en esta localidad y los reportes para las costas de Florida, Virginia y Carolina del Norte.

\section{Agradecimientos}

Los autores agradecen a Sara Quiroz-Barroso y Hermann D. Bermúdez por la revisión crítica al manuscrito original. Nuestra gratitud para Torrey Nyborg por su apoyo en las labores de prospección y recolección durante la visita al área de estudio; a Patricia Padilla Ávila por la determinación del nanoplancton calcáreo; al personal del Laboratorio Universitario de Geoquímica Isotópica (LUGIS). Instituto de Geofísica, UNAM: Teodoro Hernández Treviño y Vianney Meza García.

\section{Referencias}

Akers, W.H., 1972, Planktonic Foraminifera and biostratigraphy of some Neogene formations Northern Florida and Atlantic Coastal Plain: Tulane Studies in Geology and Paleontology, 9, 139.

Akers, W.H., 1974, Age of Pinecrest Beds, south Florida: Tulane Studies in Geology and Paleontology, 11, 119-120. 
Akers, W.H., 1979, Planktonic foraminifera and calcareous nanoplankton biostratigraphy of the Neogene of Mexico: Tulane studies in Geology and Paleontology, 15, 1-32.

Arkhangelsky, A.D., 1912, Upper Cretaceous deposits of east European Russia: Materialien zur Geologie Russlands, 25, 1-631.

Ayala-Garduño, L., 2003, Análisis tafonómico y Paleoambiental del Conglomerado de Plan del Río, Municipio de Emiliano Zapata Veracruz: México, Facultad de Ciencias, Universidad Nacional Autónoma de México, Tesis de Licenciatura, 55 p.

Berggren, W.A., 1969, Paleogene biostratigraphy and planktonic Foraminifera of Northern Europe: International Conference on Planktonic Microfossils, 1, Geneva, 1967 Proceedings, 1, 121-260.

Blow, W.H., 1969, Late middle Eocene to Recent planktonic foraminiferal biostratigraphy: Proceedings of the First International Conference on Planktonic Microfossils, 1, 199-421.

Bold, W.A. Van Den, 1978, Distribution of Tertiary and Quaternary ostracoda in Central America and Mexico, en Ferrusquia-Villafranca, I. (ed.), Conexiones terrestres entre Norte y Sudamérica: Boletín del Instituto de Geología, Universidad Nacional Autónoma de México, 101, 114-137.

Bramlette, M.N., Riedel, W.R., 1954, Stratigraphic value of discoasters and some other microfossils related to Recent coccolithophores: Journal of Paleontology, 28(4), 385-403.

Bramlette, M.N., Sullivan, F.R., 1961, Coccolithophorids and related nannoplankton of the Early Tertiary in California: Micropaleontology, 7(2), 129-188.

Brönnimann, P., Stradner, H., 1960, Die Foraminiferen und Discoasteridenzoen von Kuba und ihre interkontinentale Korrelation: Erdöl Zeitschrift für Bohr- und Foerdertechnik, 76, 364-369.

Bukry, D., Bramlette, M.N., 1969, Some new and stratigraphically useful calcareous nannofossils of the Cenozoic: Tulane Studies in Geology, 7, 131-142.

Canu, F., Bassler, R.S., 1920, North American Early tertiary Bryozoa: United States National Museum, Smithsonian Institution Bulletin, 106, 1-879.

Canu, F., Bassler, R.S., 1928, Fossil and Recent Bryozoa of the Gulf of Mexico region: Proceedings of the United States National Museum, 72, 1-199.

Castillo-Tejero, C., 1955, Bosquejo estratigráfico de la Cuenca Salina del Istmo de Tehuantepec: Boletín de la Asociación Mexicana de Geólogos Petroleros, 7, 173-212.

Cohen, K.M., Finney, S.C., Gibbard, P.L., Fan, J.-X., 2013, updated, The ICS International Chronostratigraphic Chart. Episodes 36, 199-204.

Contreras, V.H., 1956, Reseña de la geología del sureste de México (Excursión C-7) en Geología general de la Sierra Madre Oriental entre México D.F. y Córdoba Veracruz, depósitos continentales y volcánicos del Cenozoico superior y sedimentos marinos del Mesozoico y Cenozoico Campos petroleros de la Cuenca de Veracruz. Obras hidráulicas del Río Papaloapan. Campos petroleros y azufreros del Istmo de Tehuantepec. Geomorfología de la Península de Yucatán. Visitas a las zonas arqueológicas Mayas, 20th International Geological Congress, México: Boletín de la Asociación Mexicana de Geólogos Petroleros, 39-122.

Cushman, J.A., 1918, Some Pliocene and Miocene foraminifera of the coastal plain of the United States: United States Geological Survey Bulletin, 676, 1-100.

Dall, W.H., 1903, Contributions to the Tertiary fauna of Florida, with especial reference to the Miocene Silex beds of Tampa and the Pliocene beds of the Caloosahatchee River. Part 6: Transactions of the Wagner Free Institute of Science of Philadelphia, 3(6), 1219-1654.

Deflandre, G., 1942, Coccolithophoridés fossiles d'Oranie. Genres Schyphosphaera Lohmann et Thorosphaera Ostenfeld: Société d'Histoire Naturelle de Toulouse, Bulletin, 77, 125-137.

Deflandre, G., Fert, C., 1954, Observations sur les coccolithophoridés actuels et fossiles en microscopie ordinaire et électronique: Annales de Paléontologie, 40, 115-176.

d'Orbigny, A., 1826, Tableau méthodique de la classe des Céphalopodes: Annales des Sciences Naturelles, 1st Series, 7, 245-314.
Gardet, M., 1955, Contribution à l'étude des coccolithes des terrains néogènes de l'Algérie: Publications du Service de la Carte Géologique de l'Algérie (Nouvelle Série), 5, 477-550.

Gardner, J.R., 1947, The Molluscan Fauna of the Alum Bluff Group of Florida: United States Geological Survey Professional Paper, 142(F), 1-433.

Gibson, J.B., 1936, Estratigrafía y tectónica de la zona costera del Golfo entre 19³4' latitud norte y el Río Coatzacoalcos, Veracruz: Boletín de la Sociedad Geológica Mexicana, 9(5), 271-289.

Graham, A., 1994, Neogene palynofloras and terrestrial paleoenvironments in North Latin America (en línea): Estados Unidos, United States Geological Survey, actualizado 28 de febrero 2001, disponible en <http://pubs.usgs.gov/of/1994/of94-023/08_Graham.html>, consultado junio 2012.

Gran, H.H., Braarud, T., 1935, A quantitative study of the phytoplankton in the Bay of Fundy and the Gulf of Maine (including observations on hydrography, chemistry and turbidity): Journal of the Biological Board of Canada, 1(5), 279-467.

Hastings, A.B., 1947, Notes on Polyzoa (Bryozoa).-III. On some species of Cellaria, with remarks on G. Busk's grouping of the species of this genus: Annals and Magazine of Natural History, Series 11, 217-241.

Hay, W.W., Mohler, H.P., Wade, M.E., 1966, Calcareous nannofossils from Nal'chik (northwest Caucasus): Eclogae Geologicae Helvetiae, 59(1), 379-399.

Hay, W.W., Mohler, H.P., Roth, P.H., Schmidt, R.R., Boudreaux, J.E., 1967, Calcareous nannoplankton zonation of the Cenozoic of the Gulf Coast and Caribbean-Antillean area, and transoceanic correlation: Transactions of the Gulf Coast Association of Geological Societies, 17, 428-480.

Hazel, J.E., 1971, Ostracode biostratigraphy of the Yorktown Formation (upper Miocene and lower, Pliocene) of Virginia and North Carolina: Geological Survey Professional Paper, 704, 1-13.

Herrera-Anduaga, Y., 1979, Cheilostomata (Bryozoa) de la Formación Agueguexquite: México, Facultad de Ciencias, Universidad Nacional Autónoma de México, Tesis de Licenciatura, 53 p.

Herrera-Anduaga, Y., 1983, Les Bryozaires du Pliocène du Mexique et leur environnement sédimentaire: Universidad Claud Bernard, Lyon, Tesis doctoral, $146 \mathrm{p}$.

Kamptner, E., 1948, Coccolithen aus dem Torton des Inneralpinen Wiener Beckens: Anzeiger, Österreichische Akademie der Wissenschaften, Mathematische-Naturwissenschafliche Klasse, Wien, 157, 1-16.

Kamptner, E., 1963, Coccolithineen-Skelettreste aus Tiefseeablagerungen des Pazifischen Ozeans: Annalen des Naturhistorischen Museums in Wien, 66, 139-204.

Kier, P. M., 1972, Upper Miocene Echinoids from the Yorktown Formation of Virginia and their environmental significance: Washington, Smithsonian Institution Press, Smithsonian Contributions to Paleobiology, 13, 40 p.

Lancis-Sáez, C., 1998, El nanoplancton calcáreo de las cuencas Neógenas orientales de la Cordillera Bética: España, Universidad de Alicante, Tesis doctoral, $419 \mathrm{p}$.

Laboratorio Universitario de Geoquímica Isotópica (LUGIS), 2011, Introducción a la Geoquímica isotópica (en línea): México, disponible en <http://www.geofisica.unam.mx/laboratorios/ universitarios/lugis/pdf/introduc-GI.pdf>, consultado Junio 2013.

López-Ramos, E., 1979, Geología de México, Tomo III: México, Edición escolar, $445 \mathrm{p}$.

Martini, E., 1965, Mid-Tertiary calcareous nannoplankton from Pacific deep-sea cores: Colston Papers, 17, 393-411.

Martini, E., 1971, Standard Tertiary and Quaternary calcareous nannoplankton zonation, en Proceedings of the II Planktonic Conference, 1970, Rome, Tecnoscienza, 739-785.

Martini, E., Bramlette, M.N., 1963, Calcareous nannoplankton from the experimental Mohole drilling: Journal of Paleontology, 37(4), 845-855.

McArthur, J.M., Howarth, R.J., Bailey, T.R., 2001, Strontium isotope stratigraphy: Lowess Version 3 Best-fit to the marine Sr-isotope curve for 0 to $509 \mathrm{Ma}$ and accompanying look-up table for deriving numerical age: Journal of Geology, 109, 155-170. 
McLean, J.D., 1951, McLean Card Catalogue of American Foraminifera UNIT No.2, (Diagnostic larger Foraminifera of the Heterostegina zone and some smaller forms): Virginia, McLean Paleontology laboratory, Cards and Illustrations, AA171430.

Means, G.H., 2010, Alum Bluff: Florida, Southeastern Geological Society Guidebook 51 disponible en $<\mathrm{http}: / /$ segs.org/>., consultado (Junio 2013, Enero 2015).

Murray, G., Blackman, V.H., 1898, On the nature of the Coccospheres and Rhabdospheres: Philosophical Transactions of the Royal Society of London (B), 190(1): 427-441.

Okada, H., Bukry D., 1980, Supplementary modification and introduction of the code numbers to the low latitude Coccolith biostratigraphy zonation (Bukry, 1973, 1975): Marine Micropaleontology, 5, 321325.

Parker,W.K., Jones,T.R., Baley, J.W., Pourtales, F.L.,1865, On some foraminifera from the North Atlantic and Arctic Oceans, including Davis Straits and Baffin's Bay: Philosophical Transactions of the Royal Society, 155, 325-441.

Perrilliat, M.C., 1960, Moluscos del Mioceno de la Cuenca Salina del Istmo de Tehuantepec, México: Paleontología Mexicana, 8, 1-38.

Perrilliat, M. C., 1963, Moluscos de la Formación Agueguexquite (Mioceno Medio) del Istmo de Tehuantepec, México: Paleontología Mexicana, 14, 1 - 45.

Perrilliat, M.C., 1972, Monografía de los Moluscos del Mioceno medio de Santa Rosa, Veracruz, México, Parte I (Gasterópodos: Fissurellidae a Olividae): Paleontología Mexicana, 32, 1-130.

Perrilliat, M.C., 1973, Monografía de los Moluscos del Mioceno medio de Santa Rosa, Veracruz, México, Parte II (Gasterópodos: Mitridae a Terebridae): Paleontología Mexicana, 35, 1-97.

Perrilliat, M.C., 1974a, Monografía de los Moluscos del Mioceno medio de Santa Rosa, Veracruz, México: Paleontología Mexicana, Parte III (Gasterópodos: Pyramidellidae a Siphonariidae), 37, 1-46.

Perrilliat-Montoya, M.C., 1974b, Monografía de los Moluscos del Mioceno medio de Santa Rosa, Veracruz, México. Parte IV; (Escafópodos: Dentaliidae, Siphonodentaliidae): Paleontología Mexicana, 37, 51-64.

Perrilliat, M.C., 1976, Monografía de los Moluscos del Mioceno medio de Santa Rosa, Veracruz, México. Parte V. (Pelecípodos: Nuculidae a Limidae): Paleontología Mexicana, 42, 1-53.

Perrilliat, M.C., 1977, Monografía de los Moluscos del Mioceno medio de Santa Rosa, Veracruz, México. Parte VI. (Pelecípodos: Lucinidae a Solecurtidae): Paleontología Mexicana, 43, 1-60.

Perrilliat, M.C., 1984, Monografía de los Moluscos del Mioceno medio de Santa Rosa, Veracruz, México. Parte VII. (Pelecípodos: Dreissenidae a Verticordiidae: Paleontología Mexicana, 48, 1-88.

Perrilliat, MC., Flores-Guerrero, P., 2011, Moluscos de la Formación -Agueguexquite (Plioceno inferior) de Coatzacoalcos, Veracruz, México: Revista Mexicana de Ciencias Geológicas. 28 (3), 379-397.

Petróleos Mexicanos (PEMEX), 1956, Correlation Chart (excursión C-7, mapa) en Geología General de la Sierra Madre Oriental entre México D.F. y Córdoba Veracruz depósitos continentales y volcánicos del Cenozoico superior y sedimentos marinos del Mesozoico y Cenozoico. Campos petroleros de la Cuenca de Veracruz. Obras hidráulicas del Río Papaloapan. Campos petroleros y azufreros del Istmo de Tehuantepec. Geomorfología de la Península de Yucatán. Visitas a las zonas arqueológicas Mayas, 20th International Geological Congress, México: Boletín de la Asociación Mexicana de Geólogos Petroleros, Asociación Mexicana de Geólogos Petroleros.

Petuch, E.J., 1991, New gastropods from the Plio-Pleistocene of southwestern Florida and the Everglades Basin: W.H. Dall Paleontological Research Center, Special Publication 1, 63 p.

Pineda-Salgado, G., Perrilliat, M.C., Vega, F.J., 2011, Depredación de moluscos de la Formación Agueguexquite, Plioceno inferior, Región Coatzacoalcos, Veracruz (resumen) en XII Congreso Nacional de Paleontología: Puebla, 111.

Romen, F.L., 1955, Bosquejo Geológico de la Provincia del Papaloapan, Estado de Veracruz, México: Boletín de la Asociación de Geólogos Petroleros, 7 (1-2), 1-68.
Sáenz-Pita, M.R., López-Palomino, I., 2012, Agueguexquite, Formación: Léxico estratigráfico de México (en línea), México, disponible en $<$ http://mapserver.sgm.gob.mx/lexico/Agueguexquite.pdf $>$, consultado septiembre de 2014.

Sansores, De J.C., Flores-Covarrubias, C., 1972, Foraminíferos bentónicos del Terciario Superior de la Cuenca Salina del Istmo de Tehuantepec, México: Instituto Mexicano del Petróleo, 534.

Say, T., 1830, American Conchology: Or descriptions of the shells of North America. Illustrated by colored figures from original drawings executed from nature, Classic Reprint Series, $430 \mathrm{p}$.

Servicio Geológico Mexicano (SGM), 2000, Carta Geológica-Minera de Minatitlán E15-7, 1:250000 Veracruz, Oaxaca y Tabasco (en línea), disponible en http://mapserver.sgm.gob.mx/cartas_impresas/ productos/cartas/cartas250/geologia/103 E15-7GM.html,>, consultado noviembre 2012-mayo 2013.

Servicio Geológico Mexicano (SGM), 2002, Carta Geológica-Minera de Veracruz E14-3,1:250000, Veracruz Puebla y Tlaxcala (en línea), disponible en: <http://mapserver.sgm.gob.mx/cartas impresas/productos/cartas/cartas250/geologia/97_E14-3GM.html>, consultado noviembre 2012-mayo 2013.

Servicio Geológico Mexicano (SGM), 2004, Carta Geológica-Minera de Coatzacoalcos E15-1-4, 1:250000, Veracruz, Oaxaca y Tabasco (en línea), disponible en $<\mathrm{http} / /$ mapserver.sgm.gob.mx/cartas impresas/productos/cartas/cartas250/geologia/102_E15-1GM. html $>$, consultado noviembre de 2012-mayo de 2013.

Sowerby II, G.B., 1850, Descriptions of new species of fossil shells found by J.S. Heniker: Proceedings of the Geological Society of London, 6, 44-53.

Tan, S.H., 1927, Discoasteridae incertae sedis: Proceedings of the Koninklijke Nederlandse Akademie van Wetenschappen: Section of Sciences, 30, 411-419.

Tchegliakova, N., Sarmiento, G., Guerrero, J., 1997, Bioestratigrafía y Paleoecología de los foraminíferos bentónico de la Formación Chipaque y el Grupo Guadalupe, Turoniano-Maastrichtiano del Piedemonte Llanero de los Andes Colombianos: Geología Colombiana, 22, 103-119.

Thalman, H.E., 1934, Miocene Agueguexquite Formation in the Isthmus of Tehuantepec Region (abstract): Proceedings of the Geological Society of America, 116.

Theodoridis, S., 1984, Calcareous nannofossil biostratigraphy of the Miocene and revision of the helicoliths and discoasters: Utrecht Micropaleontological Bulletin, 32, 1-271.

Thomas F.C., 2005, Oligocene benthic foraminifera from the Paleogene Wenonah Canyon, Scotian Shelf - normal versus canyon assemblages: Atlantic Geology, 41(1), 1-16.

Tucker, H.I., Wilson, D., 1933, A second contribution on the Neogene paleontology of South Florida: Bulletins of American Paleontology, $18(66), 1-21$.

Vega, F.J., Nyborg, T., Coutiño, M.A., Solé, J., Hernández-Monzón, O., 2009, Neogene crustacean from southeastern México: Bulletin of the Mizunami Fossil Museum, 35, 51-69.

Waller, T.R., 2011, Neogene Paleontology of the Northern Dominican Republic. 24. Propeamussiidae and Pectinidae (Mollusca: Bivalvia: Pectinoidea) of the Cibao Valley: Bulletins of American Paleontology, 381, 1-204.

Wallich, G.C., 1877, Observations on the coccosphere: Annals and Magazine of Natural History, 19, 342-350.

Weber-van Bosse, A., 1901, Études sur les algues de l'Archipel Malaisien III. Note préliminaire sur les résultats algologíques de l'expédition du Siboga: Annales du Jardin Botanique de Buitenzorg, 17(2), 126-141.

Young, J.R., Bown, P.R., Lees J.A. (eds), Nannotax3 website. International Nannoplankton Association. Actualizado Abril 21, 2014. Disponible en http://ina.tmsoc.org/Nannotax3, consultado en (enero 2015).

Manuscrito recibido: Marzo 5, 2015

Manuscrito corregido recibido: Abril 28, 2015

Manuscrito aceptado: Mayo 3, 2015 